\title{
Response to letter to the Editor from Dr. Carbillon on our article about first pregnancy characteristics, genetics, and breast density
}

\author{
Lee Ann Prebil ${ }^{1}$
}

Received: 18 March 2015 / Accepted: 21 March 2015/Published online: 28 March 2015

(C) Springer International Publishing Switzerland 2015

\section{Dear Editor,}

My co-authors and I would like to thank Dr. Carbillon for his comments on our article about first pregnancy characteristics, genetics, and breast density. We found a modification in the association between pregnancy induced hypertension (PIH) and breast density (measured as percent fibroglandular volume, or \%FGV) by specific vascular endothelial growth factor (VEGF) (rs3025039) and insulinlike growth factor receptor-1 (IGFR1) (rs2016347) gene variants. We appreciate the additional detail Dr. Carbillon added to the discussion of PIH, VEGF, and the risk of breast cancer. The various lines of evidence pointing to the potential role of VEGF and an elevated anti-angiogenic response in the pathophysiology of breast cancer merit additional focused research. Replication of our findings of an interaction between inherited IGFR and VEGF gene variants in other populations would bolster confidence in the overall association, and indeed such studies are being planned. In addition, molecular studies examining the distribution of VEGF and IGFR1 gene variants associated with protein expression levels in women with a known history of PIH, prior breast density measurements, and subsequent diagnosis of specific breast cancer subtypes would be most informative and would help further advance our understanding of the mechanistic interaction of these risk factors.

On behalf of the MWS Research Group.

Lee Ann Prebil

lprebil@marincounty.org

1 Marin County Department of Health and Human Services, 3240 Kerner Blvd., San Rafael, CA 94901, USA 